\title{
Role of Automated Breast Ultrasound System (ABUS) as Screening Tool in Comparison To Mammogram in Detect of Different Breast Lesions
}

\author{
Hanan M. Arafa, Ahmed M. Bassiouny, Marwan A. Mohammed \\ Department of Radiodiagnosis, Faculty of Medicine, Ain Shams University \\ Corresponding author: Marwan A. Mohammed, E-mail: Marwan_figo@hotmail.com, Mobile: +201008463441
}

\begin{abstract}
Background: Mammography is an effective randomized controlled trial-proven method for reducing mortality due to breast cancer. However, the sensitivity of mammography depends on breast density. The current supplemental screening options include breast ultrasonography (US) and magnetic resonance imaging (MRI). Automated breast ultrasound system (ABUS) is an option proposed to overcome the time-consuming and costly nature of handheld, physician-performed whole-breast US (WBUS).

Objectives: To evaluate the utility of automated breast ultrasound system (ABUS) in detection of different breast lesions especially in dense breast in comparison to mammogram.

Patients and Method: It is prospective study included 25 women outreached for digital mammography or handheld ultrasound examination at the general Egyptian hospitals. Women have no specific age group.

Results: The use of ABUS with the mammogram shows significant increased frequency of detection of positive benign lesions in ACR class $\mathrm{C}$ and $\mathrm{D}$ in comparison to class $\mathrm{A}$ and $\mathrm{B}$ and insignificance malignant lesions detection.

Conclusion: ABUS reflects a promising modality in breast imaging however appears to be on a par with mammogram in terms of diagnostic quality.
\end{abstract}

Keywords: Thin Film Transistors - Terminal Ductal Lobular Unit - Triple Negative - Ultrasound.

\section{INTRODUCTION}

Mammography is an effective randomized controlled trial-proven method for reducing mortality due to breast cancer. A recent study showed $43 \%$ reduction among women participating in a national screening program. However, the sensitivity of mammography depends on breast density. Studies on women with dense breasts have demonstrated a sensitivity of less than $50 \%$. More recently, more than $50 \%$ of women younger than 50 years and at least one-third aged over 50 years have been found to have dense breast tissue. The current supplemental screening options include breast ultrasonography (US) and magnetic resonance imaging (MRI). US-based screening technologies may offer lower cost and wider availability than MRI. However, bilateral whole-breast screening using handheld US (HHUS) is time consuming, operator dependence, nonreproducibility, and time required by the radiologist to perform the exams. ABUS screening is an option proposed to overcome the time-consuming and costly nature of handheld, physician-performed whole-breast US (WBUS) ${ }^{(1)}$

Both ABUS and handheld ultrasound exhibited high sensitivity (both 100\%) and high specificity (95.0\%, and $85.0 \%$, respectively). In addition, ABUS had a higher diagnostic accuracy $(97.1 \%)$ than handheld ultrasound $\left(91.4 \%\right.$ ) for breast neoplasms ${ }^{(2)}$

Dense breast tissue obscures tumors on mammograms, making it difficult for radiologists to read and interpret the exams. This inaccuracy has resulted in an increased number of false positives and biopsies, and has increased healthcare costs and patient anxiety. For this reason recently, research and development in ultrasound are demonstrating that it is a technology that can lend itself to breast diagnostic imaging. Automated Breast Ultrasound an alternative to traditional hand-held ultrasounds for supplemental use. Like traditional ultrasound, ABUS uses high-frequency sound waves targeted at the breast, but the scans provide physicians with a 3-D volumetric image of the entire breast. These 3-D images are more beneficial to women within the dense breast population because they allow radiologists the ability to check the breast from a variety of angles and offer a better interpretation. ABUS exams are also much shorter than traditional ultrasound, with some systems taking as little as seven minutes to perform a bilateral exam - less than half the time of some traditional ultrasounds. Because the transducer used in ABUS automatically scans the breast, the operator dependency is greatly reduced ${ }^{(3)}$

\section{AIM OF THE WORK}

The aim of this study to evaluate the ABUS machine as non X-ray hazardous tool in early detection of different breast lesion.

\section{PATIENTS AND METHODS}

Type of study: This study is a prospective analysis.

Ethical and approval statements: Ethics committee of faculty of medicine, Ain shams university has approved the study protocol. 
Study population: The study included women presenting to women health unite for screening especially dense breast women and women who already had unknown breast lesion by mammogram and come for supplementary ultrasound. Cases had been supplied by different Egyptian hospitals.

\section{Inclusion Criteria:}

All women especially dense breast women undergo breast screening. All women with positive mammographic finding and undergo supplementary ultrasound.

\section{Exclusion Criteria:}

There is no exclusion criteria.

Sample size: 25 patients.

\section{Study tools:}

\section{A- Preliminary study:}

All patients have undergone handheld U/S and/or mammogram study.

\section{B- Examination using ABUS.}

All patients were submitted to the following: Demographic and clinical data collection: Including patient's name, age, marital status and phone number, duration of illness, past history, family history and provisional diagnosis.

Equipments: Mammographic examination was performed using a device developed by GE Healthcare allowing dual-energy CEDM acquisitions (Senographe 2000 D full field digital mammography Essential GE Healthcare). It used a current fullfield digital mammography system using a flat panel detector with Csl absorber, field size 19x23, del pitch of $100 \mathrm{~mm}$, image matrix size $1,914 \times 2,294$ (Senographe DS). ABUS examination was performed using a device developed by GE Healthcare allowing transmitting of ultrasound waves by high frequency probe (7-13 MHZ) through the breast tissue with specific software and hardware adaptations for acquisition and image processing.

Technique of Digital Mammography: Standard views medio-lateral-oblique and craniocauda1 views were taken for all patient.

Technique of ABUS: Standard views anterio-posterior (AP), Lateral and medial views were taken for each breast with ability to added additional views in some cases like upper quadrants and axillary tail views.

\section{RESULTS}

Table (1): Age of the patients.

\begin{tabular}{|l|c|}
\hline \multicolumn{1}{|c|}{ Age of the patients } & No. of the patients (25) \\
\hline Mean \pm SD & $43.40 \pm 9.08$ \\
Range & $29-69$ \\
$<40$ yrs & $11(44.0 \%)$ \\
$\geq 40$ yrs & $14(56.0 \%)$ \\
\hline
\end{tabular}

Table (2): ACR Density of the breast.

\begin{tabular}{|l|c|c|}
\hline $\begin{array}{c}\text { ACR Density of the } \\
\text { breast }\end{array}$ & No. of the patients & \% \\
\hline A & 4 & $16.0 \%$ \\
\hline B & 8 & $32.0 \%$ \\
\hline C & 9 & $36.0 \%$ \\
\hline
\end{tabular}

Table (3): Detected lesions by mammogram and correlated BIRADS.

\begin{tabular}{|l|l|c|}
\hline \multicolumn{2}{|c|}{ Lesions } & No. of the patients (25) \\
\hline \multirow{2}{*}{ Mammogram } & Negative & $8(32.0 \%)$ \\
& Positive & $17(68.0 \%)$ \\
\hline \multirow{4}{*}{ Mammogram } & 1 & $8(32.0 \%)$ \\
BIRADS & 2 & $7(28.0 \%)$ \\
& 3 & $3(12.0 \%)$ \\
& 4 & $4(16.0 \%)$ \\
& 5 & $3(12.0 \%)$ \\
& Mean \pm SD & $2.48 \pm 1.42$ \\
& Range & $1-5$ \\
\hline
\end{tabular}

Table (4): Detected lesions by ABUS and correlated BIRADS.

\begin{tabular}{|l|l|c|}
\hline \multicolumn{2}{|c|}{ Lesions } & No. of the patients (25) \\
\hline \multirow{2}{*}{ ABUS } & Negative & $5(20.0 \%)$ \\
& Positive & $20(80.0 \%)$ \\
\hline \multirow{5}{*}{ ABUS BIRADS } & 1 & $5(20.0 \%)$ \\
& 2 & $8(32.0 \%)$ \\
& 3 & $5(20.0 \%)$ \\
& 4 & $4(16.0 \%)$ \\
& 5 & $3(12.0 \%)$ \\
& Mean \pm SD & $2.68 \pm 1.31$ \\
& Range & $1-5$ \\
\hline
\end{tabular}

Table (5): Comparison between detected lesions and correlated BIRADS by mammogram and ABUS.

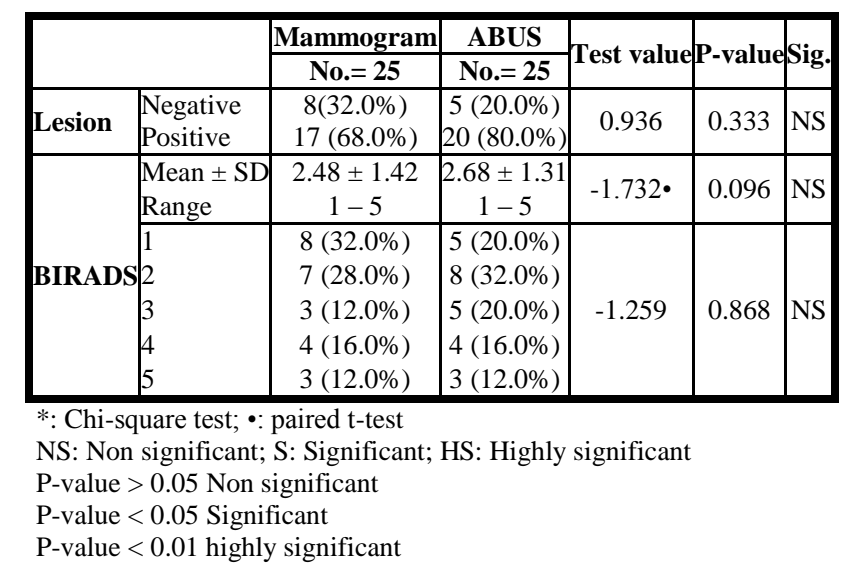


Table (6): Comparison between detected lesions and correlated BIRADS by mammogram and ABUS.

\begin{tabular}{|c|c|c|c|c|c|c|c|c|}
\hline \multicolumn{2}{|c|}{} & \multicolumn{2}{|c|}{$\begin{array}{c}\text { Negative } \\
\text { Mammogram }\end{array}$} & \multicolumn{2}{|c|}{ Mammogram } & \multirow{2}{*}{ Test value* } & P-value & Sig. \\
\cline { 3 - 7 } \multicolumn{2}{|c|}{} & No. & $\%$ & No. & $\%$ & & & \\
\hline \multirow{2}{*}{ ABUS } & Negative & 5 & $62.5 \%$ & 0 & $0.0 \%$ & 13.281 & 0.000 & HS \\
& Positive & 3 & $37.5 \%$ & 17 & $100.0 \%$ & & & \\
\hline
\end{tabular}

*: Chi-square test

NS: Non significant; S: Significant; HS: Highly significant

$\mathrm{P}$-value $>0.05$ Non significant

$\mathrm{P}$-value $<0.05$ Significant

$\mathrm{P}$-value $<0.01$ highly significant

Table (7): Ratio of sensitivity and specificity of ABUS.

\begin{tabular}{|l|c|c|c|c|c|}
\hline & Sensitivity & Specificity & $+\mathbf{P V}$ & $\mathbf{- P V}$ & Accuracy \\
\hline ABUS & $100.0 \%$ & $62.5 \%$ & $85.0 \%$ & $100.0 \%$ & $88.0 \%$ \\
\hline
\end{tabular}

Table (8): ACR

\begin{tabular}{|c|c|c|c|c|c|c|c|}
\hline \multirow{2}{*}{ ACR } & \multicolumn{2}{|c|}{$<\mathbf{4 0}$ yrs } & \multicolumn{2}{|c|}{$>\mathbf{4 0}$ yrs } & \multirow{2}{*}{ Test value* } & P-value & Sig. \\
\cline { 2 - 7 } & No. & $\%$ & No. & $\%$ & & & \\
\hline A & 2 & $18.2 \%$ & 2 & $14.3 \%$ & & & \\
B & 1 & $9.1 \%$ & 7 & $50.0 \%$ & & & \\
C & 5 & $45.5 \%$ & 4 & $28.6 \%$ & & 0.149 & NS \\
D & 3 & $27.3 \%$ & 1 & $7.1 \%$ & & & \\
\hline
\end{tabular}

*: Chi-square test

NS: Non significant; S: Significant; HS: Highly significant

P-value $>0.05$ Non significant

$\mathrm{P}$-value $<0.05$ Significant

$\mathrm{P}$-value $<0.01$ highly significant

Table (9): Comparison between detected lesions and correlated BIRADS by mammogram with patient's ages.

\begin{tabular}{|c|c|c|c|c|c|c|}
\hline & $<40 \mathrm{yrs}$ & $>=40 \mathrm{yrs}$ & \multirow{2}{*}{$\begin{array}{c}\text { Test } \\
\text { value }\end{array}$} & \multirow{2}{*}{$\begin{array}{c}\text { P- } \\
\text { value }\end{array}$} & \multirow{2}{*}{ Sig. } \\
\hline & & No. $=11$ & No. $=14$ & & & \\
\hline \multirow{7}{*}{$\begin{array}{l}\text { Mammogram } \\
\text { BIRADS }\end{array}$} & Mean \pm SD & $1.73 \pm 1.01$ & $3.07 \pm 1.44$ & 2026 & 0015 & $\mathrm{~S}$ \\
\hline & Range & $1-4$ & $1-5$ & $-2.0200^{\circ}$ & & \\
\hline & 1 & $6(54.5 \%)$ & $2(14.3 \%)$ & \multirow{5}{*}{$6.206^{*}$} & \multirow{5}{*}{0.184} & \multirow{5}{*}{ NS } \\
\hline & 2 & $3(27.3 \%)$ & $4(28.6 \%)$ & & & \\
\hline & 3 & $1(9.1 \%)$ & $2(14.3 \%)$ & & & \\
\hline & 4 & $1(9.1 \%)$ & $3(21.4 \%)$ & & & \\
\hline & 5 & $0(0.0 \%)$ & $3(21.4 \%)$ & & & \\
\hline \multirow{2}{*}{ Mammogram } & Negative & $6(54.5 \%)$ & $2(14.3 \%)$ & \multirow{2}{*}{$4.588 *$} & \multirow{2}{*}{0.032} & \multirow{2}{*}{$S$} \\
\hline & Positive & $5(45.5 \%)$ & $12(85.7 \%)$ & & & \\
\hline
\end{tabular}

*: Chi-square test; $\bullet$ : Independent t-test

NS: Non significant; S: Significant; HS: Highly significant

P-value $>0.05$ Non significant

P-value $<0.05$ Significant

$\mathrm{P}$-value $<0.01$ highly significant
Table (10): Comparison between detected lesions and correlated BIRADS by ABUS with patient's ages.

\begin{tabular}{|c|c|c|c|c|c|c|}
\hline & $<40 \mathrm{yrs}$ & $>=40 \mathrm{yrs}$ & \multirow{2}{*}{$\begin{array}{c}\text { Test } \\
\text { value }\end{array}$} & \multirow{2}{*}{$\begin{array}{c}\text { P- } \\
\text { value }\end{array}$} & \multirow{2}{*}{ Sig } \\
\hline & & No. $=11$ & No. $=14$ & & & \\
\hline \multirow{7}{*}{$\begin{array}{l}\text { ABUS } \\
\text { BIRADS }\end{array}$} & Mean \pm SD & $2.00 \pm 1.00$ & $3.21 \pm 1.31$ & $-2541 \bullet$ & 0.018 & $\mathrm{~S}$ \\
\hline & Range & $1-4$ & $1-5$ & & & \\
\hline & 1 & $4(36.4 \%)$ & $1(7.1 \%)$ & \multirow{5}{*}{$5.722^{*}$} & \multirow{5}{*}{0.221} & \multirow{5}{*}{ NS } \\
\hline & 2 & $4(36.4 \%)$ & $4(28.6 \%)$ & & & \\
\hline & 3 & $2(18.2 \%)$ & $3(21.4 \%)$ & & & \\
\hline & 4 & $1(9.1 \%)$ & $3(21.4 \%)$ & & & \\
\hline & 5 & $0(0.0 \%)$ & $3(21.4 \%)$ & & & \\
\hline \multirow{2}{*}{ ABUS } & Negative & $4(36.4 \%)$ & $1(7.1 \%)$ & \multirow{2}{*}{$3.287^{*}$} & \multirow{2}{*}{0.070} & \multirow{2}{*}{ NS } \\
\hline & Positive & $7(63.6 \%)$ & $13(92.9 \%)$ & & & \\
\hline
\end{tabular}

*: Chi-square test; $\bullet:$ Independent t-test

NS: Non significant; S: Significant; HS: Highly significant

P-value $>0.05$ Non significant

$\mathrm{P}$-value $<0.05$ Significant

$\mathrm{P}$-value $<0.01$ highly significant

\section{DISCUSSION}

Breast lesions remains one of the leading causes of women health problems specially breast cancer remain one of the leading causes of death in women over the age of 40 years ${ }^{(4)}$

Breast lesions screening is used to identify women with asymptomatic and mildly symptomatic lesions with the goal of enabling women to undergo less treatments that lead to better outcomes.

Mammography is the best-studied breast screening modality and the only recommended imaging tool for screening the general population of women.

Mammographic breast density is a wellestablished and strong predictor of breast cancer risk. Women with extensive breast density are at higher risk of developing breast cancer ${ }^{(5)}$.

The goals of automating breast ultrasound are: Decrease the radiologist's time per case. Produce a standardized, high quality examination that improves the conspicuity of cancers.

In contrast to HHUS, 3D automated breast ultrasound system (ABUS) has a standardized acquisition protocol that can be performed by medical personnel after short training without the need for highly trained radiologists during the examination.

Because of its capabilities, 3D ABUS enables reproducibility and can in essence 
eliminate the investigator-dependent and nonstandardized documentation (6). These are characteristics that could make 3D ABUS a very useful addition to the diagnostic breast screening.

ABUS systems can automatically scan the entire breast in a standard manner with optimized settings (imaging presets) for volume acquisition based on the estimated size of the breast (A is smallest size; D+ is largest size), the system applies imaging parameters during acquisition based on the estimated size of the breast and automatically send all the images to an ABVS workstation ${ }^{(7)}$.

\section{CONCLUSION}

ABUS is a computer-based system for performing and recording ultrasound of the entire breast. It has a workstation that allows physicians to review ultrasound images from different angles with reconstructions in the axial and coronal planes and three dimensional. The transducer is attached to a computerguided mechanical arm, and images acquired in longitudinal rows, acquiring transverse images.

ABUS provides advantages of: High diagnostic accuracy. Better lesion size prediction. Operator-independence. Visualization of the whole breast. Ability to do examination in women with contraindication to mammogram.

The use of ABUS with mammography improved the accuracy of breast lesions detection, callback rates, and confidence in callbacks. In conclusion, ABUS reflects a promising modality in breast imaging however appears to be on a par with hand-held ultrasound in terms of diagnostic quality.

\section{REFERENCES}

1. Hak Hee Kim and Joo Hee Cha (2015): Current status of automated breast ultrasonography. Ultrasonography, 34(3): 165-172.

2. Xi L, Jianwei $W$ and Feng $H$ (2012): Analysis of eighty-one cases with breast lesions using automated breast volume scanner and comparison with handheld ultrasound. European Journal of Radiology,81 (5): 873-878.

3. Jacob JMD (2012): Dense Breast Tissue: Supplemental Screening. Imaging Technology News, 11: 46-49.

4. Yang $\mathbf{T}$, Liang $\mathrm{H}$, Chouc $\mathrm{C}$ Huang $\mathrm{J}$, and Pan H (2013): Adjunctive Digital Breast Tomosynthesis in Diagnosis of Breast Cancer. Biomed Res., 597253.
5. Sleeba T, Subapradha A, Ramachandran $M$ and Krishnaswami M (2013): Role of dualenergy contrast-enhanced digital mammography as a problem-solving tool in dense breasts: a case report. The Indian journal of radiology \& imaging, 23(3): 219.

6. Berg A (2003): Mammography screening: are women really giving informed consent? (Counterpoint). J Nat! Cancer Inst., 95(20): 1511-2.

7. Wang H, Jianga $Y$, Zhua Q, Zhanga J, Daia $Q$, Liua H, Lai X, Sunb Q (2012): "Differentiation of benign and malignant breast lesions: A comparison between automatically generated breast volume scans and handheld ultrasound examinations"; European Journal of Radiology, 81: 3190- 3200. 